\title{
BLAST System: Different Decoders with Different Antennas
}

\author{
Pargat Singh Sidhu ${ }^{1}$, Amit Grover $^{2 *}$, Neeti Grover ${ }^{3}$ \\ 1(Research Scholar, Department of Electronics and Communication Engineering, Shaheed Bhagat Singh State \\ Technical Campus, Moga Road (NH-95), Ferozepur-152004, India) \\ $2 *$ (Assistant Professor, Department of Electronics and Communication Engineering, Shaheed Bhagat Singh \\ State Technical Campus, Moga Road (NH-95), Ferozepur-152004, India. \\ 3(Assistant Professor, Department of Applied sciences, Shaheed Bhagat Singh State Technical Campus, Moga \\ Road (NH-95), Ferozepur-152004, India)
}

\begin{abstract}
Wireless communication technology has shown that the application of multiple antennas at both transmitter and receiver sides improve the possibility of high data rates through multiplexing or to improve performance through diversity compared to single antenna systems. In this article, the BER performance of MMSE and Maximum Likelihood (ML) -Vertical Bells Lab Layered Space Time Architecture (V-BLAST) with different modulation techniques such as BPSK and QPSK, in Rayleigh flat fading channel has been considered. Further, the comparison of these modulation techniques with different multiple antenna configurations in Rayleigh channel is carried out. It has been concluded that both the decoders perform better using BPSK modulation scheme as compare to QPSK modulation scheme and we obtain more optimal results for $1 \times 4$ antennas for both MMSE V-BLAST and ML-V-BLAST system in Rayleigh channel. While comparing the ML and MMSE-VBLAST for $2 \mathrm{X} 2$ antenna configurations, the better results has been obtained for ML-VBLAST System as compare to MMSE V-BLAST System in Rayleigh Channel.
\end{abstract}

Keywords: Binary Phase Shift Key (BPSK), Bit Error Rate (BER), Minimum Mean Square error (MMSE), Maximum Likelihood (ML), Vertical Bell Laboratories Layered Space-Time (V-BLAST)

\section{INTRODUCTION}

Wireless communication system with multi-antenna arrays has been a field of intensive research on the last years [10]. The use of multiple antennas at both the transmitter and the receiver sides can drastically improve the channel capacity and data rate [8]. The study of the performance limits of MIMO system [3] becomes very important since it will give lot ideas in understanding and designing the practical MIMO systems [4]. Vertical-Bell Laboratories Layered Space-Time (V-BLAST) Architecture and first practical implementation of this architecture on MIMO wireless communications to demonstrate a spectral efficiency as high as 40bits/s/Hz in real time in the laboratory [6]. Many schemes have been proposed to explode the high spectral efficiency of MIMO channels, among which V-BLAST [6] is relatively simple and easy to implement and can achieve a large spectral efficiency. Detection process [5] mainly involves three operations: Interference Suppression, interference cancellation and Optimal Ordering [12].V-BLAST algorithm [6] integrates both linear and non-linear algorithms presented in the interference nulling and interference cancellation respectively, in an independent identically distributed Flat fading Rayleigh channel [1] with ' $\mathrm{N}$ ' transmitting antennas and ' $\mathrm{M}$ ' receiving antennas [12].

\section{MIMO CHANNEL MODEL}

Let us consider a communication system with ' $\mathrm{N}$ ' number of transmitting antennas and ' $\mathrm{M}$ ' number of receiving antennas in Ricean Flat Fading channel [1] shown in Fig. 1.

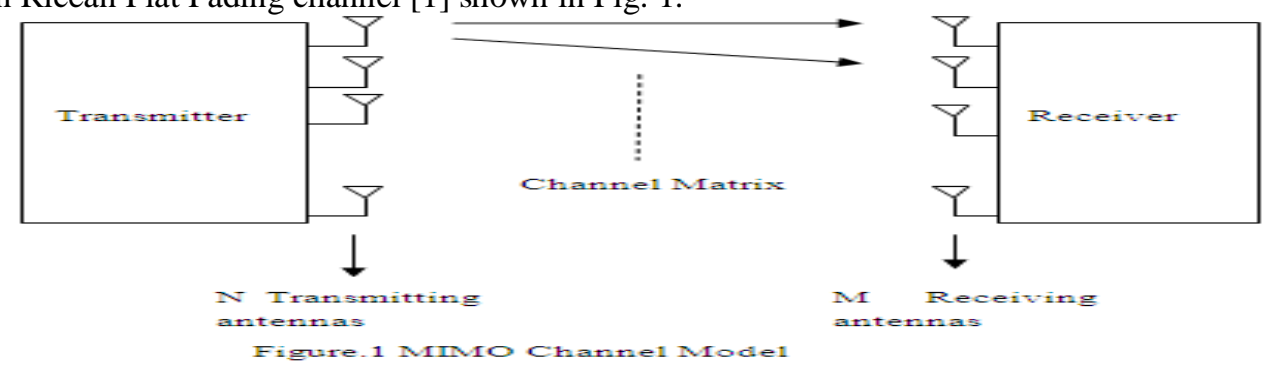

The sampled baseband representation of signal is given by

$$
\mathrm{y}=\mathrm{Hx}+\mathrm{n}
$$


And the complex baseband representation of signal [7] is given by

$$
\mathrm{y}=\sqrt{ }(\mathrm{P} / \mathrm{M}) \mathrm{Hx}+\mathrm{n}
$$

Where $\mathrm{y} \varepsilon \mathrm{C}^{\mathrm{NX} 1}$ is the received signal vector, $\mathrm{x} \varepsilon \mathrm{C}^{\mathrm{MX} 1}$ is the transmitted signal vector with zero mean and unit variance, $\mathrm{P}$ is the total transmit power, $\mathrm{H} \varepsilon \mathrm{C}^{\mathrm{NXM}}$ is the channel response matrix with possibly correlated fading coefficients. In order to access the performance of V-BLAST in correlated channel, we adopted a correlationbased channel model which is expressed as

$$
\mathrm{H} \sim \mathrm{R}_{\mathrm{Rx}}^{1 / 2} \mathrm{H}_{\mathrm{W}}\left(\mathrm{R}_{\mathrm{Tx}}^{1 / 2}\right)^{\mathrm{T}}
$$

where $\mathrm{x} \sim \mathrm{y}$ denotes that $\mathrm{x}$ and $\mathrm{y}$ are identical in distribution, $\mathrm{R}_{\mathrm{Rx}}$ and $\mathrm{T}_{\mathrm{Tx}}$ are the normal correlation distribution matrices at the $R_{x}$ and transmitter $\left(T_{x}\right)$ respectively, and $H_{W} \varepsilon C^{N X M}$ contains independent identically distributed complex Gaussian entries with zero mean and unit variance.

\section{FADING CHANNEL}

Fading is used to describe the rapid fluctuations of the amplitudes, phases or multipath delays of a radio signal over a short period of time or travel distance, so that large scale path loss effect may be ignored.

\subsection{RAYLEIGH FADING CHANNEL}

The fading effect is usually described statistically using the Rayleigh distribution. The amplitude of two quadrature Gaussian signals follows the Rayleigh distribution whereas the phase follows a uniform distribution. The probability distribution function (PDF) of a Rayleigh distribution is given by

$$
p(r)=\left\{\begin{array}{cl}
\frac{r}{\sigma^{2}} \exp \left(\frac{-r^{2}}{2 \sigma^{2}}\right) & (0 \leq r \leq \infty \\
0 & (r<0)
\end{array}\right\}
$$

Where $\sigma$ is the RMS (amplitude) value of the received signal and $\sigma^{2}$ is the average power.

\section{V-BLAST-SYSTEM-MODEL}

A high-level block diagram of a V-BLAST system [5] is shown in
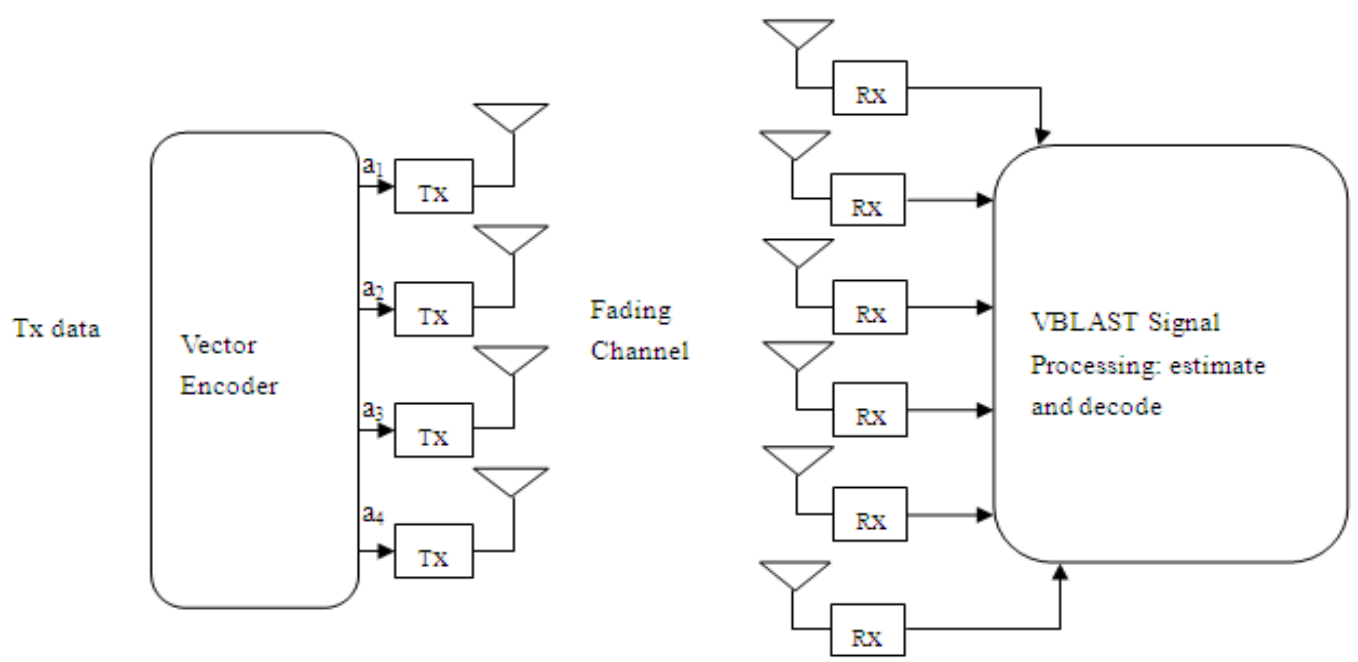

Figure.2 V-BLAST MIMO System Model

\subsection{ENCODER}

For simplicity, we base our explanation on Figure 2. Suppose the number of transmitting antennas is $\mathbf{M}_{T}$ and the number of receiving antennas is $\mathbf{M}_{R}$. For example we take QAM modulation, transmitters 1 to $\mathbf{M}_{T}$ operate co-channel at symbol rate $1 / \mathrm{T}$ symbols, with synchronized symbol timing. This collection of transmitters constitutes a vector drawn from a QAM constellation. Receivers 1 to $M_{R}$ are individually conventional QAM receivers. The receivers also operate co-channel, each receiving the signals radiated from all $M_{T}$ transmit antennas. Flat fading is assumed and the matrix channel transfer function is $H_{R}{ }_{R}{ }_{M}{ }_{T}$ where $h_{i j}$ is the complex transfer function from transmitter $\mathrm{j}$ to receiver $\mathrm{i}$ and $\mathrm{M}_{\mathrm{T}} \mathrm{X} \mathrm{M}_{\mathrm{R}}$. We assume that the transmission is organized in bursts of $\mathrm{L}$ symbols and that the channel time variation is negligible over the $\mathrm{L}$ symbol periods, comprising a burst, and that the channel is estimated accurately using training symbols embedded in each burst 
vector $\mathrm{i}$

Let $\mathrm{a}=\left(\begin{array}{llll}a_{1} & \mathrm{a}_{2} & \ldots \ldots \mathrm{a}_{\mathrm{M}}\end{array}\right)^{\mathrm{T}}$ denote the vector of transmit symbols. Then the corresponding received $\mathrm{M}_{\mathrm{R}}$

Where $\mathrm{n}$ is a wide sense stationary (WSS) noise vector [11].

$$
\mathrm{r}_{1}=\mathrm{Ha}+\mathrm{n}
$$

\subsection{DECODER}

The decoder needs to demodulate the symbols on the received vector. If channel encoding is used, then the demodulated symbols need to be buffered until the whole block can be decoded. Otherwise, the demodulation can be done immediately.

\section{DECODING ALGORITHM FOR V-BLAST SYSTEM}

One approach to a lower complexity design of the receiver is to use a "divide-and-conquer" strategy instead of decoding all symbols jointly. First, the algorithm decodes the strongest symbol. Then, canceling the effects of this strongest symbol from all received signals, the algorithm detects the next strongest symbol. The algorithm continues by canceling the effects of the detected symbol and the decoding of the next strongest symbol until all symbols are detected. The optimal detection order is from the strongest symbol to the weakest one. This is the original decoding algorithm [5] of V-BLAST preset. It only works if the number of receive antennas is more than the number of transmit antennas, that is $\mathrm{M} \times \mathrm{N}$. Decoding Algorithm of V-BLAST is shown in Figure.3

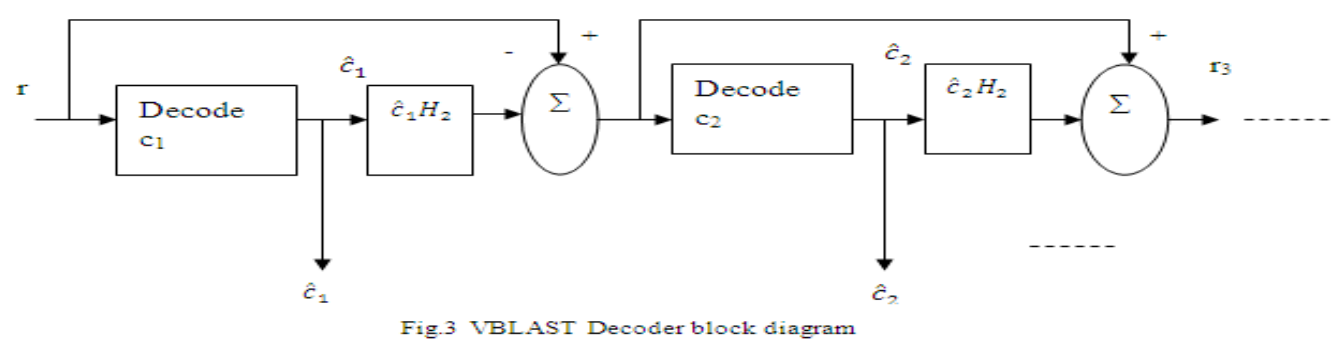

The algorithm includes three steps which includes ordering, interference cancellation and Interference nulling

\subsection{ORDERING}

In decoding the first symbol, the interference from all other symbols is considered as noise. After finding the best candidate for the first symbol, the effects of this symbol in the entire receiver equations are canceled [12].

\subsection{INTERFERENCE CANCELLATION}

At stage $\mathrm{n}$ of the algorithm, when $\mathrm{c}_{\mathrm{n}}$ is being detected, symbols $\mathrm{c}_{1}, \mathrm{c}_{2}, \mathrm{c}_{\mathrm{n}-1}$ have been already detected. Let us assume a perfect decoder that is the decoded symbols $\left(\hat{c}_{1}, \widehat{c}_{2}\right.$ and $\left.\hat{c}_{n-1}\right)$ are the same as the transmitted symbols $c_{1}, c_{2}, c_{n-1}$. One can subtract $\sum_{\mathrm{i}=1}{ }^{\mathrm{N}-1} c_{i} H_{i}$ from the received vector $\mathrm{r}$ to derive an equation that relates remaining undetected symbols to the received vector

$$
\begin{gathered}
r_{n}=r-\sum_{i=1}^{n-1} c_{i} H_{i}+N, \\
r_{n}=\sum_{i=n}^{N} c_{i} H_{i}+N, \quad n=1,2 \ldots . N-1
\end{gathered}
$$

In fact, by using induction in addition to the convention $r_{1}=r$, one can show that

$$
r_{n+1}=r_{n}-c_{n} H_{n}, \quad n=1,2,3 \ldots . . N-1
$$

Therefore, at the $\mathrm{n}^{\text {th }}$ stage of the algorithm after detecting the $\mathrm{nth}$ symbol as $\widehat{c}_{n}$, its effect is canceled from the equations by

$$
r_{n+1}=r_{n}+\hat{c}_{n} H_{n}
$$

This interference cancelation is conceptually similar to DFE [2].

\subsection{INTERFERENCE NULLING}

Interference nulling is the process of detecting $c_{n}$ from $r_{n}$ by first removing the effects of undetected symbols. Basically, in this step the nth symbol is detected by nulling the interference caused by symbols $\mathrm{c}_{\mathrm{n}+1}$, $\mathrm{c}_{\mathrm{n}+2}, \mathrm{c}_{\mathrm{N}}$. Like any other interference suppression problem, there are many different methods to detect a symbol in the presence of interference. 


\section{ML-V-BLAST-DECODER}

The ML receiver [12] performs optimum vector decoding and is optimal in the sense of minimizing the error probability. ML receiver is a method that compares the received signals with all possible transmitted signal vectors which is modified by channel matrix $\mathrm{H}$ and estimates transmit symbol vector $\widehat{\mathrm{C}}$ according to the Maximum Likelihood principle [12], which is shown as:

$$
\widehat{\mathrm{C}}=\underset{\mathrm{C}}{\operatorname{argmin}} \llbracket \mathrm{r}-\mathrm{C}^{\prime} \mathrm{H} \rrbracket_{\mathrm{F}}^{2}
$$

where $\mathrm{F}$ is the Frobenius norm. Expanding the cost function using Frobenius norm given by

$$
\begin{gathered}
\hat{\mathrm{C}}=\underset{\widehat{\mathrm{C}}}{\operatorname{argmin}}\left[\operatorname{Tr}\left[\left(\mathrm{r}-\mathrm{C}^{\prime} \mathrm{H}\right)^{\mathrm{H}} \cdot\left(\mathrm{r} 00-\mathrm{C}^{\prime} \mathrm{H}\right)\right\rceil\right] \\
\widehat{\mathrm{C}}=\underset{\widehat{\mathrm{C}}}{\operatorname{argmin}}\left[\operatorname{Tr}\left[\mathrm{r}^{\mathrm{H}} \cdot \mathrm{r}+\mathrm{H}^{\mathrm{H}} \cdot \mathrm{C}^{\prime \mathrm{H}} \cdot \mathrm{C}^{\prime} \cdot \mathrm{H}-\mathrm{H}^{\mathrm{H}} \cdot \mathrm{C}^{\prime \mathrm{H}} \cdot r-\mathrm{r}^{\mathrm{H}} \cdot \mathrm{C}^{\prime} \cdot \mathrm{H}\right\rceil\right]
\end{gathered}
$$

Considering $\mathrm{r}^{\mathrm{H}} . \mathrm{r}$ is independent of the transmitted codeword so can be rewritten as

$$
\widehat{\mathrm{C}}=\underset{\widehat{\mathrm{C}}}{\operatorname{argmin}}\left[\operatorname{Tr}\left[\mathrm{H}^{\mathrm{H}} \cdot \mathrm{C}^{\mathrm{H}} \cdot \mathrm{C}^{\prime} \cdot \mathrm{H}\right]-2 \cdot \operatorname{Real}\left(\operatorname{Tr}\left[\mathrm{H}^{\mathrm{H}} \cdot \mathrm{C}^{\mathrm{H}} \cdot \mathrm{r}\right]\right)\right]
$$

Equation (12) can be re-written for multiple receivers as shown in equation (13).

$$
\widehat{\mathrm{C}}=\underset{\widehat{\mathrm{C}}}{\operatorname{argmin}}\left\lceil\sum_{\mathrm{m}=1}^{\mathrm{M}_{\mathrm{R}}}\left[\mathrm{H}_{\mathrm{m}}^{\mathrm{H}} \cdot \mathrm{C}^{\prime} \mathrm{H} \cdot \mathrm{C}^{\prime} \cdot \mathrm{H}_{\mathrm{m}}-2 \cdot \operatorname{Real}\left(\mathrm{H}_{\mathrm{m}}^{\mathrm{H}} \cdot \mathrm{C}^{\prime} \cdot \mathrm{r}_{\mathrm{m}}\right)\right]\right\rceil
$$

Where ${ }^{\mathrm{H}}$ is a Hermition operator. We can write the cost function for only one receiving antenna and then added up to achieve for $\mathrm{M}_{\mathrm{R}}$ receiving antenna.

$$
\left.\left\lceil H_{m}^{H} \cdot C^{\prime H} \cdot C^{\prime} \cdot H_{m}-2 \cdot \operatorname{Real}\left(H_{m}^{H} \cdot C^{\prime} \cdot r_{m}\right)\right]\right\rceil
$$

Where the minimization is performed over all possible transmit estimated vector symbols. Although ML detection offers optimal error performance, it suffers from complexity issues.

\section{MMSE-V-BLAST DECODER}

The MMSE receiver suppresses both the interference and noise components. This implies that the mean square error between the transmitted symbols and the estimate of the receiver is minimized. At low SNR, MMSE becomes matched filter [12].

\section{SIMULATION AND RESULTS}

The simulation has been carried out by using MATLAB 7.0 for the Bit Error Rate (BER) Performance of the ML-VBLAST System [13]. The simulation of BER performance of ML-VBLAST and MMSE-VBLAST Systems in Rayleigh flat fading channel [1] has been done using the different modulation techniques like BPSK and QPSK

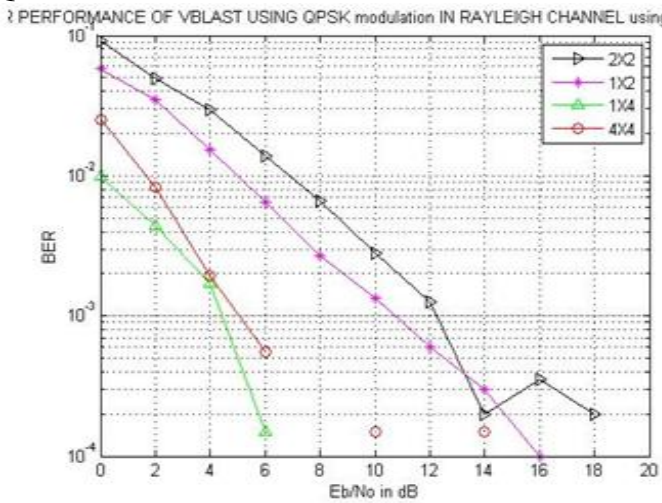

Figure.4

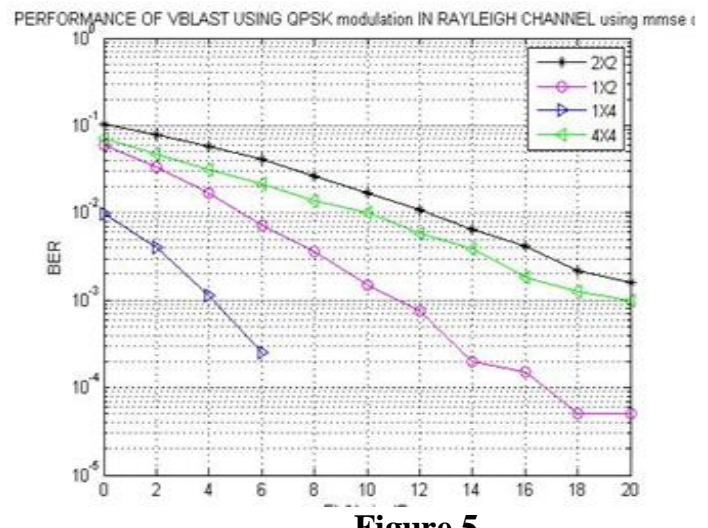

Figure.5

Figure 4 and 5 shows BER for ML-VBLAST and MMSE-VBLAST using QPSK modulation 


\begin{tabular}{|c|c|}
\hline M X N & BER \\
\hline $1 \times 4$ & .0025 \\
\hline $4 \times 4$ & 0.0050 \\
\hline $1 \times 2$ & 0.019 \\
\hline $2 \times 2$ & 0.01 \\
\hline
\end{tabular}

Table 1

\begin{tabular}{|c|c|}
\hline M X N & BER \\
\hline $1 \times 4$ & .000398 \\
\hline $4 \times 4$ & 0.050 \\
\hline $1 \times 2$ & 0.025 \\
\hline $2 \times 2$ & 0.079 \\
\hline
\end{tabular}

Table 2

Table 1 and Table 2 shows BER for ML-VBLAST and for MMSE-VBLAST using QPSK modulation in Rayleigh Channel

In Figure 4 and 5, we get an optimal result for $1 \times 4$ antenna configuration than another antenna configurations using QPSK modulation in Rayleigh channel. From table.1, At SNR=2dB, 1X4 antenna configuration has the minimum value (0.0025) of BER than another configuration. From Table.2, At SNR=2dB $1 \mathrm{X} 4$, antenna configuration has the minimum value (0.000398) than other antenna configuration. So we can say that $1 \times 4$ configuration has best performance than another antenna configurations using QPSK modulation in Rayleigh channel both for ML and MMSE.

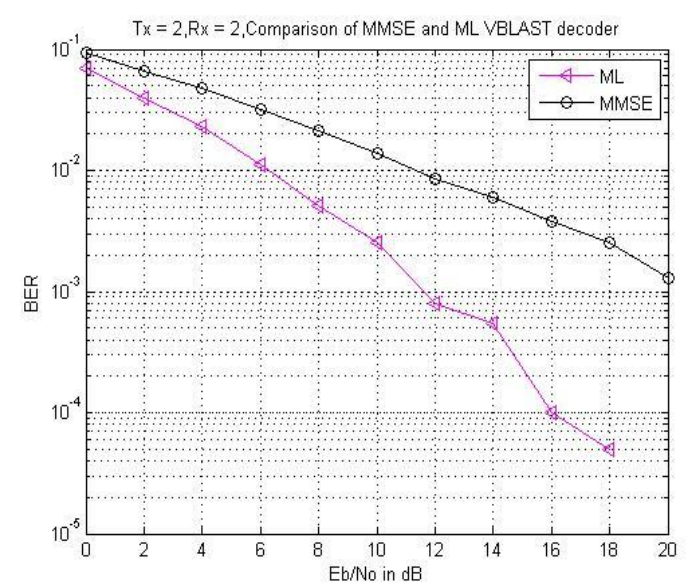

Figure.6

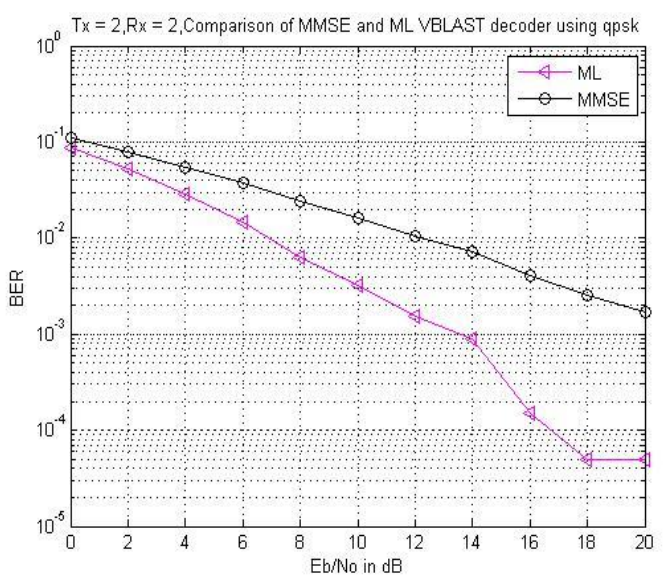

Figure.7

Figure 6 and 7 shows Comparison of ML and MMSE-VBLAST using BPSK and QPSK modulation

Finally, the comparison of ML-VBLAST and MMSE-VBLAST decoders in 2X2 antenna configuration using BPSK and QPSK modulations has been considered. In figure 6 at SNR $=6 \mathrm{~dB}$, the value of BER for MLVBLAST decoder is 0.001 and for MMSE-VBLAST is 0.01 and In figure 7 at SNR $=6 \mathrm{~dB}$, the value of BER for ML-VBLAST decoder is 0.001 and for MMSE-VBLAST is 0.031 which shows that ML-VBLAST decoder gives the better result than MMSE-VBLAST decoder for both BPSK and QPSK modulation techniques.

\section{CONCLUSIONS}

Finally, it has been concluded that by keeping more number of receiving antennas than transmitting antennas, a better BER performance has been achieved and more number of errors can be removed. Keeping more number of transmitting antennas than receiving antennas results in a worst BER performance and removes a few errors. By comparing ML-VBLAST and MMSE-VBLAST decoders in Rayleigh channel for both BPSK and QPSK modulations a better performance in case of ML-VBLAST decoder has been achieved.

\section{REFERENCES}

[1]. R.U. Nabar, H.Boleskei and A.J. Paulraj (2005)," Diversity and outage performance in Space Time Block Coded Rician MIMO Channels" IEEE Trans. Wireless Commun. Vol.4, pp.1102-1111.

[2]. G.Ginis and J.M.Cioffi (2001), "On the relationship between V-BLAST and GDFE," IEEE Communications letters, vol. 5, pp. 364-366.

[3]. R. U. Nabar A. J. Paulraj, D. A. Gore and H. Bolcskei (2004), "An overview of MIMO communications - a key to gigabit wireless," Proceedings of the IEEE, vol. 92, no. 2, pp. 198-218. 
[4]. A.Paulraj and R.J.Heath (2001), "Characterization of MIMO Channels for Spatial Multiplexing Systems "IEEE International Conference on Communications, vol.2, no.11-14, pp-591-595.

[5]. G.D.Golden, G.J.Foschini, R.A. Valenzuela, and P.W.Wolniasky (1999), "Detection algorithm and initial laboratory results using the V-BLAST space-time communication architecture," Electron Lett., vol.35, no.1, pp.1415.

[6]. P.Wolniosky, G.J.Foschini, G.D.Golden and R.A. Valenzuela (1998)," V-BLAST: An Architecture for realizing very high data rates over rich scattering wireless channel" URSI International Symposium on Signals, Systems and Electronics, ISSSE 98.

[7]. Choi, J, Yu,H., and Lee, Y.H.(2005), "Adaptive MIMO decision Feedback Equalization for Receivers with time varying channels", IEEE transaction on signal processing, vol.55, No. 7, pp.3405-3416

[8]. I.E. Telatar (1999), "Capacity of multi-antenna Gaussian channels, "European Transactions on Telecommunications, vol. 10, no.6, pp.585-595.

[9]. M.Varanasi and T.Guess (1997), "Optimum decision feedback multiuser equalization with successive decoding achieves the total capacity of the Gaussian multiple-access channel," Conference Record of the Thirty-First Asilomar Conference on signals, Systems and computers, vol. 2, pp. 1405-1409.

[10]. X.Li, H.Huang, G.J.Foschini, and R.A.Valenzu, "Effects of Iterative Detection and Decoding on the Performance of BLAST", IEEE Global Telecommunications Conference, vol.2, pp.1061-1066, Nov 2000.

[11]. G. J. Foschini, "Layered space-time architecture for wireless communication in a fading environment using multi-element antennas," Bell-Labs Techn. J., pp. 41-59, 1996.

[12]. C.Windpassinger and RF.H Fischer, "Low-complexity need-Maximum Likelihood detection and precoding for MIMO systems" in ITW 2003, Paris, France, March 31-April-4, 2003.

[13]. A. Paulraj, R.Nabar and D.Gore, "Introduction to Space Time Wireless Communications", Cambridge University Press, May 2003.

[14]. Sukhchain Singh, Gurpreet Singh and Amit Grover (2012), "Performance Evaluation of ML-VBLAST MIMO System using various antenna configurations with Ricean and Rayleigh Channel" Innovative Systems Design and Engineering, vol. 3, no. 10, pp. 62-71.

[15]. Amit Grover, Neeti Grover (2012), "MMSE-V-BLAST Optimal-Ordering in Different Channels" IOSR Journal of Engineering, vol. 2, issue 11, pp. 51-57.

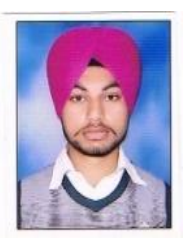

\section{BIOGRAPHY}

Pargat Singh Sidhu is pursuing his research work in the area of Electronics and Communication Engineering under the guidance of Mr. Amit Grover, Assistant Professor, Department of Electronics and Communication Engineering, Shaheed Bhagat Singh State Technical Campus, Moga road, Ferozepur, Punjab, India. Pargat Singh Sidhu received his B. Tech degree in the area of Electronics \& Communication Engineering in 2011 from LLRIET, Moga, Punjab. His area of interest includes Signal processing, MIMO systems, Wireless mobile communication.

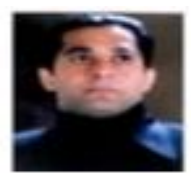

Amit Grover became a Member (M) of Association ISTE in 2006, a Senior Member (SM) of society SELCOME in September 2009, and a Project-In charge (PI) in august 2011 and in September 2012. The author place of birth is Ferozepur, Punjab, India on $27^{\mathrm{TH}}$, September 1980. The author received his M. Tech degree in Electronics and Communication Engineering from Punjab Technical University, Kapurthla, Punjab, India in 2008 and received his B. Tech degree in Electronics and Communication Engineering from Punjab Technical University, Kapurthala, Punjab, India in 2001. Currently, he is working as an Assistant Professor in Shaheed Bhagat Singh State Technical Campus, Moga road, Ferozepur, Punjab, India. He has a working experience of 11 years in teaching. The author is a reviewer of different international journals. His area of interest includes signal processing, MIMO Systems, Wireless mobile communication, High speed digital communications and 4G wireless communications.

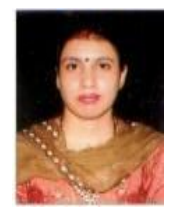

Neeti Grover received her master degree in Applied Sciences from Guru Nanak Dev University, Amritsar, and Punjab, India in 2007 and received her Bachelor's degree from Guru Nanak Dev University, Amritsar, Punjab, India in 2004. Her place of birth is Jallandhar, Punjab, India on $29^{\mathrm{TH}}$, December 1983. Currently, she is working as an Assistant Professor in the department of Applied Sciences and Humanities in Shaheed Bhagat Singh State Technical Campus (Poly Wing), Ferozpur, Punjab, India. 